Check for updates

Cite this: RSC Adv., 2017, 7, 31110

Received 28th April 2017 Accepted 1st June 2017

DOI: $10.1039 / c 7 r a 04767 c$

rsc.li/rsc-advances

\section{Simultaneously high thermal stability and ultra-fast phase change speed based on samarium-doped antimony thin films}

\begin{abstract}
Hua Zou, (D) *a Yifeng Hu, ${ }^{a}$ Xiaoqin Zhu*a and Zhitang Song ${ }^{\text {b }}$
The trade-off between crystallization speed and thermal stability has been a challenge to improve the performance of phase change memory. Herein, we propose samarium (Sm)-doped antimony (Sb) materials, in which the Sm doping influences the thermal stability to simultaneously realize high thermal stability and ultra-fast phase change speed. We show that slight Sm doping ( $<3 \%)$ can improve the crystallization temperature $\left(T_{\mathrm{c}}\right)$ up to $242{ }^{\circ} \mathrm{C}$ and their relevant 10 year data retention up to $159{ }^{\circ} \mathrm{C}$ with an ultra-fast speed of $\sim 2$ ns. The high performance of Sm doped Sb thin film was attributed to the formation of Sm-Sb bonds measured by XPS. These results suggest that the Sm doped Sb materials are promising candidates for phase change memory, and the rare-earth (RE) doping-induced improvement in performance could be extended to other chalcogenide films.
\end{abstract}

\section{Introduction}

Phase change memory (PCM) techniques based on phase change materials are derived from the extreme optical or electrical contrast between the amorphous and crystalline structural states. ${ }^{1}$ Compared to other data storage technologies, such as dynamic random access memory (DRAM) and ferroelectric memories (FeRAM), phase change materials offer fast access speed, high density storage, non-volatility, and good scalability. $^{2}$ To date, PCM is considered as a promising next generation memory, which operates via a thermally induced reversible phase transition between the amorphous and crystalline state, where the former state represents the " 0 " state (high resistance) and the latter expresses the " 1 " state (low resistance). ${ }^{3}$

Recently, $\mathrm{Ge}_{2} \mathrm{Sb}_{2} \mathrm{Te}_{5}$ (GST), sandwiched between two conduction contacts, is the most widely applied material for PCM applications due to its overall suitable properties of good stability and high speed. ${ }^{4}$ However, GST has some drawbacks: the Te element is harmful to semiconductor techniques due to its volatilization ${ }^{5,6}$ and the phase change speed $\sim 50$ ns makes it difficult for high speed PCM applications. ${ }^{7,8}$ Thus, it has been challenging to prepare materials possessing high thermal stability and ultra-high phase change speed..$^{9-11}$

Antimony ( $\mathrm{Sb}$ ) has become another choice for PCM because of the fastest speed change, which originates from growth-

${ }^{a}$ School of Mathematics and Physics, Jiangsu University of Technology, Changzhou, 213001, P. R. China.E-mail: zouhua@jsut.edu.cn; zxq@jsut.edu.cn

${ }^{b}$ State Key Laboratory of Functional Materials for Informatics, Shanghai Institute of Micro-system and Information Technology, Chinese Academy of Sciences, Shanghai 200050, China dominant crystallization behavior. ${ }^{12}$ However, pure $\mathrm{Sb}$ thin films have very low thermal stability, even if its initially deposited films possess a crystalline state., ${ }^{\mathbf{8 1 2}}$ Recently, a useful strategy for improving the thermal stability takes the doping path. ${ }^{\mathbf{1} 13}$ For example, the $\mathrm{O}, \mathrm{N}, \mathrm{SiC}^{\mathbf{1 4}}$ and $\mathrm{Si}^{15}$ doping can enhance the thermal stability of Sb-based films. However, very little study has been carried out to investigate rare earth (RE)doped chalcogenide films. In our previous study, it was found that Er can significantly improve the thermal stability of Sbbased films. For extending the RE-doped phase change materials, it is important to continue investigating the properties of other RE-doped thin films. As is known, samarium (Sm) is a typical member of the lanthanides with only slight toxicity and has several important applications, such as a catalyst ${ }^{\mathbf{1 6}}$ and luminescence center. ${ }^{17}$ In this study, we fabricated a Sm-doped $\mathrm{Sb}$ thin film and studied the thermal stability, phase change speed, and investigated the possible mechanism in detail.

\section{Experimental}

The Sm-doped Sb thin films $(1 \mathrm{~cm} \times 1 \mathrm{~cm})$ of $\mathrm{Sm}_{x} \mathrm{Sb}_{1-x}(0.005 \leq$ $x \leq 0.025$ ) were deposited on $\mathrm{SiO}_{2} / \mathrm{Si}$ (100) wafers by cosputtering the $\mathrm{Sm}$ and $\mathrm{Sb}$ targets at room temperature using magnetron sputtering. The purity of the $\mathrm{Sm}$ and $\mathrm{Sb}$ targets was 99.999\%. The base pressure in the deposition chamber was $2 \times$ $10^{-4} \mathrm{~Pa}$. Sputtering was performed under an $\mathrm{Ar}$ gas pressure of $0.3 \mathrm{~Pa}$, a flow of $30 \mathrm{SCCM}$, and power of $30 \mathrm{~W}$. The thin film thickness was set at $50 \mathrm{~nm}$ through controlling the deposition time. To ensure the uniformity of the deposition, the substrate holder was rotated at an autorotation speed of $20 \mathrm{rpm}$.

The amorphous-to-crystalline transition was investigated by in situ temperature-dependent resistance $(R-T)$ measurements 
using a TP 95 temperature controller (Linkam Scientific Instruments Ltd. Surry, UK) under an Ar atmosphere. Laser irradiation was performed on the films using a mode-locked neodymium-doped yttrium aluminum garnet laser operating at a wavelength of $532 \mathrm{~nm}$ with approximately $30 \mathrm{ps}$. The He-Ne laser with a wavelength of $632.8 \mathrm{~nm}$ was used as the probing laser. The compositions of the deposited films were determined by means of energy dispersive spectroscopy (EDS). The crystalline structures of the films were analyzed by X-ray diffraction (XRD, PANalytical, X'PERT Powder). The surface morphology of the films was examined by atomic force microscopy (AFM, FMNanoview 1000). In order to assess the chemical bonding states of the $\mathrm{Sm}$ doped $\mathrm{Sb}$ films, X-ray photoelectron spectroscopy (XPS, Thermo Scientific, K-Alpha) was carried out.

\section{Results and discussion}

Fig. 1(a) shows the resistance of the Sm doped Sb films $(x=$ $0.005,0.010,0.015,0.020$ and 0.025 ) as a function of temperature at a heating rate of $20{ }^{\circ} \mathrm{C} \mathrm{min}{ }^{-1}$. It can be clearly found that the a precipitous drop, defined as the crystallization temperature $\left(T_{\mathrm{c}}\right)$, occurs at $165{ }^{\circ} \mathrm{C}, 191{ }^{\circ} \mathrm{C}, 209{ }^{\circ} \mathrm{C}, 231{ }^{\circ} \mathrm{C}$ and $243{ }^{\circ} \mathrm{C}$ upon increasing the doping content from 0.005 to 0.025 . This result reveals that the $\mathrm{Sm}$ dopants can significantly inhibit the growth-dominant crystallization process, leading to a remarkable improvement of the thermal stability. In addition, the resistance of the amorphous and crystalline state increases upon increasing the doping content of $\mathrm{Sm}$, indicating a decrease in the writing current of their PCM devices. ${ }^{18}$
The 10 year data retentions of the Sm doped Sb thin films are shown in Fig. 1(b), which can be characterized by extrapolating the isothermal plots in the Arrheniuus equation:

$$
t=\tau \exp \left(\frac{E_{\mathrm{c}}}{K_{\mathrm{b}} T}\right)
$$

where $t, \tau, E_{\mathrm{c}}, K_{\mathrm{b}}$ and $T$ are the failure time, pre-exponential factor, activation energy, Boltzmann's constant and the absolute temperature, respectively. From the Fig. 1(b), it can be shown that the 10 year retention temperature was $92{ }^{\circ} \mathrm{C}, 109^{\circ} \mathrm{C}$, $109{ }^{\circ} \mathrm{C}, 123{ }^{\circ} \mathrm{C}$ and $156{ }^{\circ} \mathrm{C}$, and the $E_{\mathrm{c}}$ was $2.93 \mathrm{eV}, 2.87 \mathrm{eV}$, $3.02 \mathrm{eV}, 3.92 \mathrm{eV}$ and $3.64 \mathrm{eV}$, respectively. These results reveal that the Sm doped $\mathrm{Sb}$ alloys can store data much longer and safer upon Sm doping. More importantly, the films with a doping content larger than 0.015 can possess a 10 year data retention temperature higher than $125^{\circ} \mathrm{C}$, which will satisfy the requirement for PCM devices competing with NOR flash memory. ${ }^{19}$

The phase change speed is the key index for materials in data memory, which can be characterized by the change in the optical reflectivity. Fig. 2 shows the evolution of reflectivity for $\mathrm{Sm}_{0.005} \mathrm{Sb}_{0.995}$ and $\mathrm{Sm}_{0.015} \mathrm{Sb}_{0.985}$ excited by laser irradiation. When excited with a laser pulse of fluence $0.56 \mathrm{~mJ} \mathrm{~cm}^{-2}$, the reflectivity increases due to the transition from an amorphous to crystalline state, as shown in Fig. 2(a). For comparison, the reverse amorphous-to-crystalline transition was also achieved using a higher laser fluence of $1.72 \mathrm{~mJ} \mathrm{~cm} \mathrm{~cm}^{-2}$, showing a decrease in the optical reflectivity. In Fig. 2(b), the clear peaks with reverse direction may be ascribed to the $\mathrm{Sb}$ volatilization or
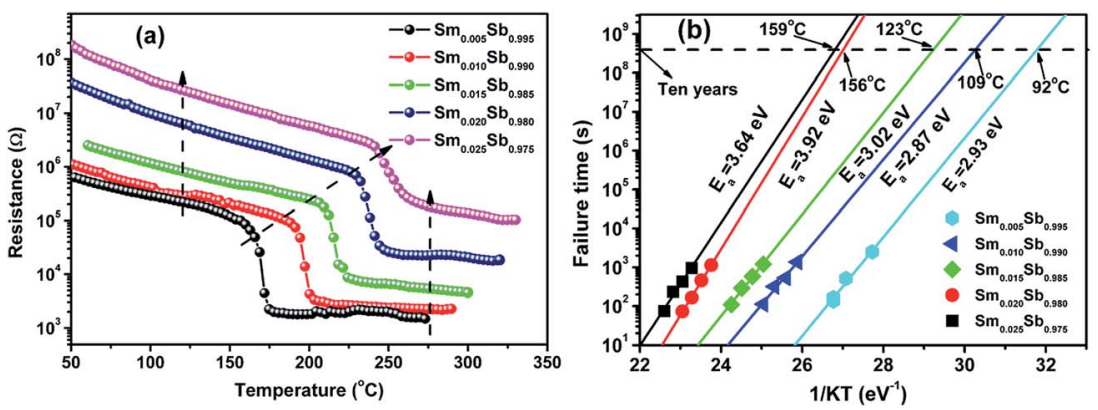

Fig. 1 (a) The sheet resistance of the Sm doped Sb films as an function of temperature with a heating rate of $20^{\circ} \mathrm{C} \mathrm{min}{ }^{-1}$. (b) Plot of "time of failure" vs. reciprocal temperature (isothermal process) showing the data retention time.
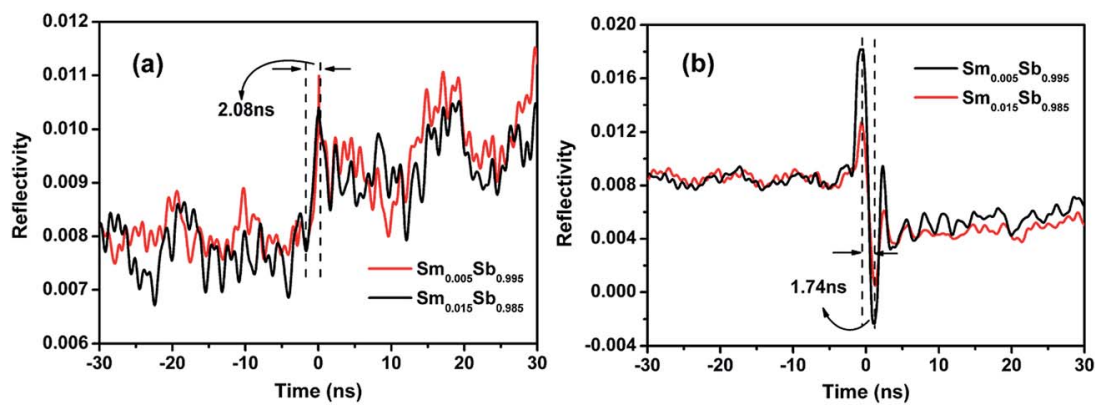

Fig. 2 Evolution of the reversible reflectivity of the $\mathrm{Sm}_{0.005} \mathrm{Sb}_{0.995}$ and $\mathrm{Sm}_{0.015} \mathrm{Sb}_{0.985}$ thin films induced by two consecutive picoseconds laser pulses with different fluence: (a) the crystallization process and (b) the amorphization process. 

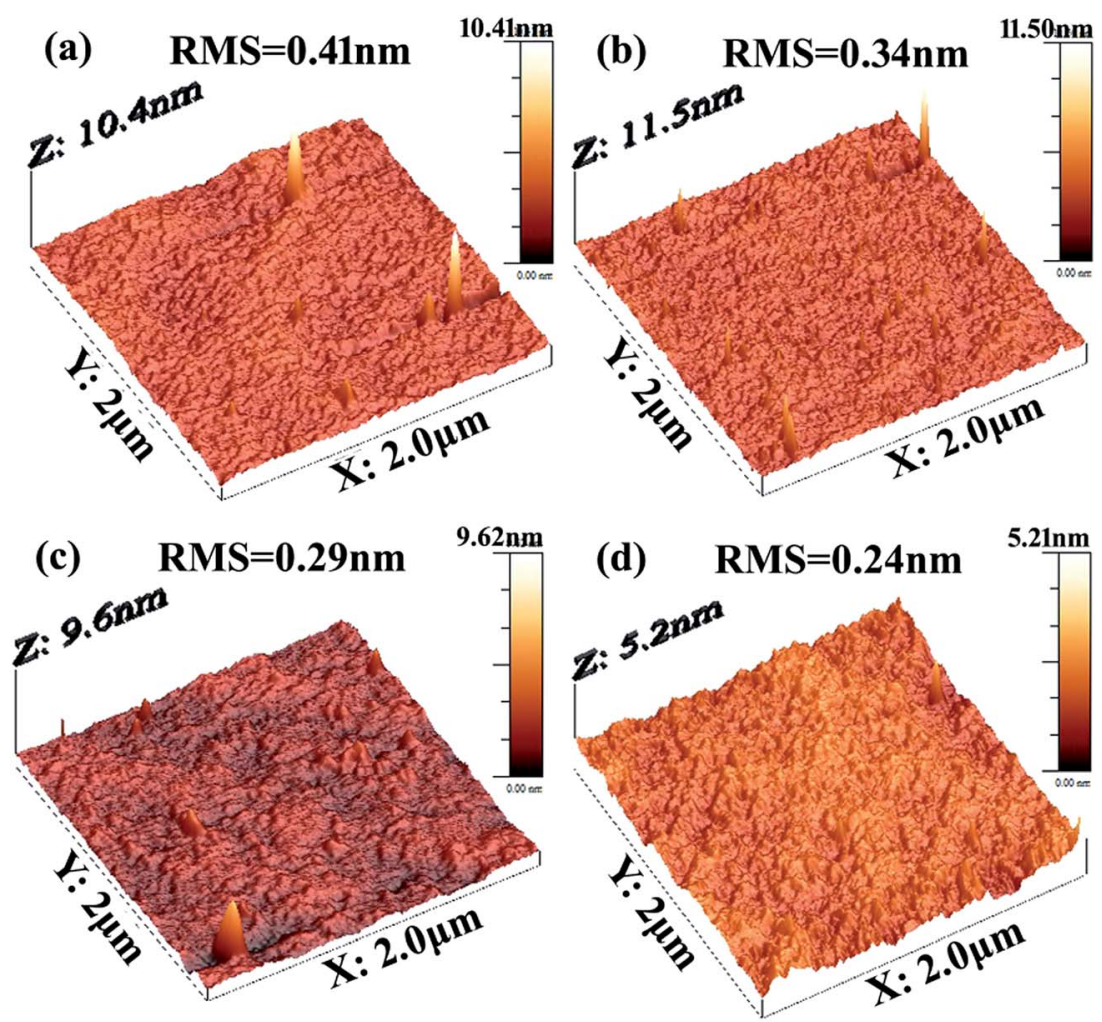

Fig. 3 AFM image of the as-deposited (a) $\mathrm{Sm}_{0.010} \mathrm{Sb}_{0.990}$, (b) $\mathrm{Sm}_{0.015} \mathrm{Sb}_{0.985}$,

(c) $\mathrm{Sm}_{0.020} \mathrm{Sb}_{0.980}$ and (d) $\mathrm{Sm}_{0.025} \mathrm{Sb}_{0.975}$

hole opening and propagation upon increasing the laser power. ${ }^{20}$ Compared with the laser fluence of GST $\left(7.31 \mathrm{~mJ} \mathrm{~cm}^{-2}\right.$ for the crystallization process and $12.31 \mathrm{~mJ} \mathrm{~cm}^{-2}$ for the amorphization process), ${ }^{21}$ the Sm doped Sb materials possess much lower laser fluence, revealing the lower power consumption for phase change. More importantly, Fig. 2 reveals that the crystallization time for the Sm doped Sb films was about 2.08 $\mathrm{ns}$, which is much shorter than GST $(\sim 17 \mathrm{~ns})^{21}$ and other known Sb-rich alloys such as SbSe $(\sim 11 \mathrm{~ns})^{22}$ and GeSb $(\sim 12 \mathrm{~ns}) \cdot{ }^{23}$ Similarly, the amorphization process has a much shorter time (about 1.74 ns) than GST ( 16 ns) or other systems. Thus, these results demonstrate that the Sm doped Sb thin films have an ultra-high phase change speed due to their growth-dominated crystallization process. ${ }^{24}$ In general, the Sb-rich materials will have fast phase change speed because of the weak $\mathrm{Sb}-\mathrm{Sb}$ bond. The doped atoms, such as Sm, will form a new bond such as $\mathrm{Sm}-\mathrm{Sb}$, which can easily break the $\mathrm{Sb}-\mathrm{Sb}$ bond. ${ }^{25} \mathrm{In}$ this study, the ultra-high phase change speed was ascribed to the growthdominated crystallization mechanism of the $\mathrm{Sb}-\mathrm{Sb}$ bond. More importantly, the slight Sm doping allows the films to still possess the massive nucleating center of $\mathrm{Sb}-\mathrm{Sb}$, keeping the ultra-high phase change speed.

The surface roughness of the films is very important for the device performance because the electric properties depend on the quality of the electrode-film interface ${ }^{26} \mathrm{Fig} .3$ shows the film surface roughness of the Sm doped Sb films observed by AFM. Generally, the root mean square (RMS) is calculated as the root mean square of the surface measured microscopic peaks and valleys, which is the key index for surface roughness. As shown in Fig. 4, the RMS of the as-deposited $\mathrm{Sm}_{0.005} \mathrm{Sb}_{0.995}$, $\mathrm{Sm}_{0.010} \mathrm{Sb}_{0.990}, \mathrm{Sm}_{0.015} \mathrm{Sb}_{0.985}, \mathrm{Sm}_{0.020} \mathrm{Sb}_{0.980}$ are 0.41, 0.34, 0.29 and $0.24 \mathrm{~nm}$, respectively. Compared with other results, such as $\mathrm{Sn}_{2} \mathrm{Se}_{3}$ (ref. 27) and $\mathrm{Sb}_{2} \mathrm{Te}_{3}{ }^{28}$ the $\mathrm{Sm}$ doped $\mathrm{Sb}$ films have a relatively low RMS value, demonstrating the excellent surface roughness for the devices performance. In addition, it is clearly found that the RMS values decrease upon increasing the content of the Sm dopant due to the impediment of the crystallization

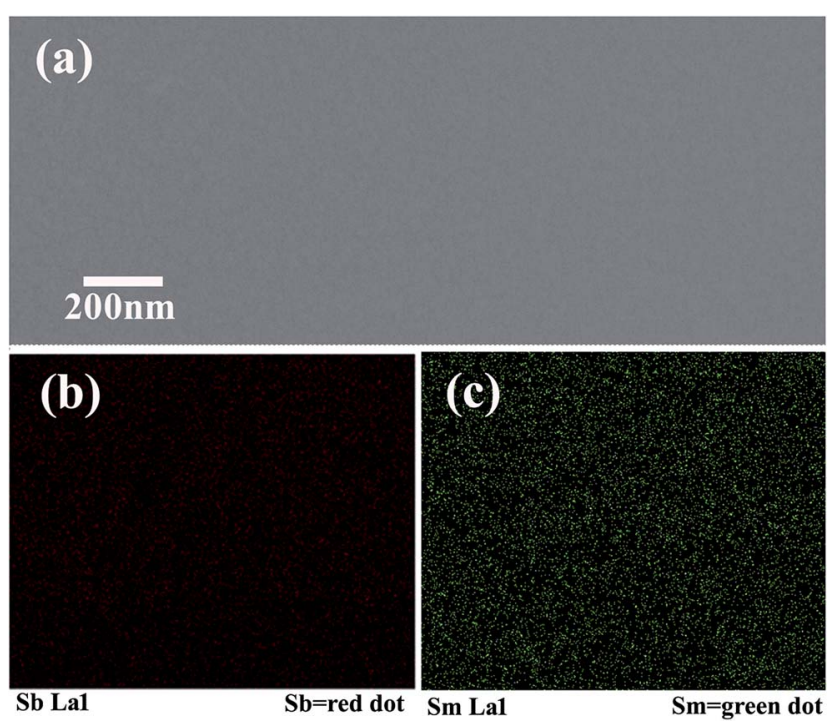

Fig. 4 (a) The surface morphology and ( $b$ and c) EDS mapping of the as-prepared $\mathrm{Sm}_{0.025} \mathrm{Sb}_{0.975}$ thin film. 

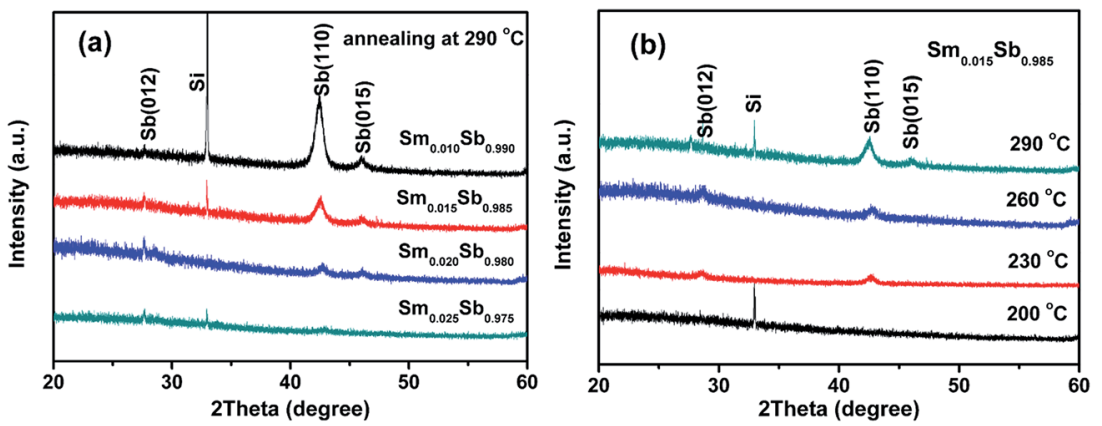

Fig. 5 (a) The XRD patterns of the $\mathrm{Sm}_{x} \mathrm{Sb}_{1-x}$ films $(x=0.010,0.015,0.020$ and $x=0.025)$ annealed at $290^{\circ} \mathrm{C}$ for $5 \mathrm{~min}$. (b) The XRD pattern of the $\mathrm{Sm}_{0.015} \mathrm{Sb}_{0.985}$ films annealed at different temperatures for $5 \mathrm{~min}$.
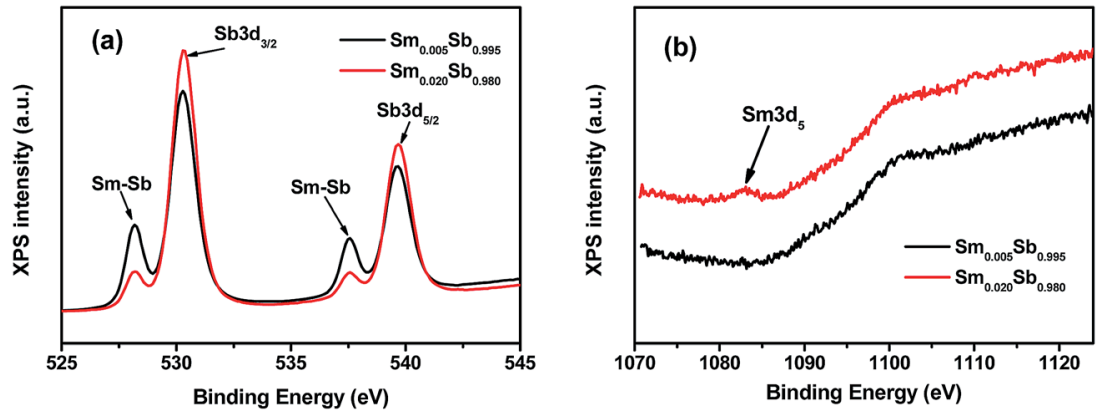

Fig. 6 (a) High resolution Sb3d and (b) high resolution Sm3d XPS spectra of the $\mathrm{Sm}_{0.005} \mathrm{Sb}_{0.995}$ and $\mathrm{Sm}_{0.020} \mathrm{Sb}_{0.980}$ thin films annealed at $290{ }^{\circ} \mathrm{C}$ for 5 min.

process by the Sm doping. On this point, the Sm dopants will be propitious to improve the performance of PCM devices.

Fig. 4(a) shows the SEM images of the as-prepared $\mathrm{Sm}_{0.025} \mathrm{Sb}_{0.975}$ thin film. It is apparent that no crystalline grains were observed in the film, also indicating the smooth amorphous state. The corresponding EDS elemental mappings are shown in Fig. 4(b) and (c). Through Fig. 4(c), it is apparent that there is a homogeneous distribution of $\mathrm{Sm}$ in the $\mathrm{Sb}$ thin film. In addition, Fig. 4(b) shows the Sb elemental homogeneous distributed over the surface. These results directly demonstrate that the $\mathrm{Sb}$ films are embedded with uniform Sm elements.

Fig. 5 shows the crystalline structures of the $\mathrm{Sm}$ doped $\mathrm{Sb}$ films characterized by XRD after annealing in an Ar atmosphere for $5 \mathrm{~min}$. As shown in Fig. 5(a), only the single hexagonal phase of $\mathrm{Sb}$ (PDF\#35-0732) was observed after annealing at $290{ }^{\circ} \mathrm{C}$. Moreover, Fig. 5(a) also indicates that the intensity of the XRD peaks significantly decreased upon increasing the Sm doping resulting in a significant increase in the $T_{\mathrm{c}}$, which is in accordance with the $R-T$ measurements. Fig. 5(b) shows the XRD patterns of the $\mathrm{Sm}_{0.015} \mathrm{Sb}_{0.985}$ film annealed at different temperatures. It can be seen that when the annealing temperature was lower than $200^{\circ} \mathrm{C}$, the film maintained an amorphous state. When the temperature was higher than $230^{\circ} \mathrm{C}$, the crystallization peaks emerged, which could be assigned to the phase transition. This result reveals that the $\mathrm{Sm}_{0.015} \mathrm{Sb}_{0.985}$ thin film can keep a stable amorphous state at $200{ }^{\circ} \mathrm{C}$, indicating excellent thermal reliability for PCM applications.
In order to comprehend the mechanism for the enhanced thermal stability, the bond environments of the doped crystalline films were measured using XPS, as shown in Fig. 6. The XPS analysis in Fig. 6(a) indicates that the $\mathrm{Sm}-\mathrm{Sb}$ bonds exists in the crystalline $\mathrm{Sm}$ doped $\mathrm{Sb}$ films, which is consistent with $\mathrm{Er}$ doped Sb films. ${ }^{29}$ Thus, the formation of some $\mathrm{Sm}-\mathrm{Sb}$ bonds during the crystallization process will increase the $E_{\mathrm{c}}$ and enhance the thermal stability. In addition, Fig. 6(a) also shows that the relative intensity of the $\mathrm{Sm}-\mathrm{Sb}$ peaks decreases upon increasing the Sm content, which may be attributed to the lower $T_{\mathrm{c}}$ with a lower amount of dopant. ${ }^{30,31}$ Fig. 6(b) indicates that some $\mathrm{Sm}-\mathrm{Sm}$ bonds exist in the $\mathrm{Sm}_{0.020} \mathrm{Sb}_{0.980}$ film only when the doping content is larger than 0.020 . This powerfully illustrates that, with slight Sm doping, the formation of the Sm-Sb bonds plays the main role in the control of thermal stability.

\section{Conclusions}

In summary, $\mathrm{Sm}$ doped $\mathrm{Sb}$ thin films were prepared via $\mathrm{RF}$ magnetron sputtering. The crystallization temperature $\left(T_{\mathrm{c}}\right)$ and 10 year data retention of the Sm doped Sb films both increase upon increasing the amount of Sm doping and are both higher than those of conventional GST films. A reversible phase transition was achieved by picosecond laser pulses, which indicated that the Sm doped Sb thin films had an ultra-high phase change speed. AFM tests revealed that the $\mathrm{Sm}_{x} \mathrm{Sb}_{1-x}$ alloys have excellent surface roughness, and the value of the RMS decreased 
upon increasing the amount of Sm doping. EDX mapping showed that the Sm elements were homogenously distributed in the $\mathrm{Sb}$ thin films. The XRD measurements reveal that the Sm doped $\mathrm{Sb}$ films have a single $\mathrm{Sb}$ phase and also proved the significant improvement via Sm doping. The XPS analyses show the improvement in the device performance was due to the Sm$\mathrm{Sb}$ bonding. Thus, the outstanding properties of simultaneous high thermal stability and ultra-fast phase change speed make the $\mathrm{Sm}$ doped $\mathrm{Sb}$ thin films promising candidates for PCM applications.

\section{Acknowledgements}

This study was supported by the Natural Science Foundation of Jiangsu Province (No. BK20151172) and Changzhou Sci \& Tech Program (No. CJ20159049, CJ20160028), and sponsored by the Qing Lan Project.

\section{References}

1 T. H. Lee, D. Loke and S. R. Elliott, Adv. Mater., 2015, 27, 5477-5483.

2 X. Zhou, J. Kalikka, X. Ji, L. Wu, Z. Song and R. E. Simpson, Adv. Mater., 2016, 28, 3007-3016.

3 D. Lencer, M. Salinga, B. Grabowski, T. Hickel, J. Neugebauer and M. Wuttig, Nat. Mater., 2008, 7, 972-977.

4 S. Raoux and D. Ielmini, Chem. Rev., 2009, 110, 240-267.

5 Y. Lu, S. Song, X. Shen, G. Wang, L. Wu, Z. Song, B. Liu and S. Dai, J. Alloys Compd., 2014, 586, 669-673.

6 Y. Gu, T. Zhang, Z. Song, Y. Liu, B. Liu and S. Feng, Appl. Phys. A: Mater. Sci. Process., 2010, 99, 205-209.

7 P. C. Chang, C. C. Chang, S. C. Chang and T. S. Chin, J. NonCryst. Solids, 2014, 383, 106-111.

8 H. Zou, X. Zhu, Y. Hu, Y. Sui, W. Wu, J. Xue, L. Zheng and Z. Song, CrystEngComm, 2016, 18, 6365-6369.

9 X. Zhou, L. Wu, Z. Song, F. Rao, K. Ren, C. Peng, S. Song, B. Liu, L. Xu and S. Feng, Appl. Phys. Lett., 2013, 103, 072114.

10 K. Ren, F. Rao, Z. Song, C. Peng, J. Li, L. Wu, B. Liu and S. Feng, Appl. Phys. Lett., 2013, 103, 093111.

11 S. Y. Lee, H. K. Kim, J. H. Kim, J. S. Roh and D. J. Choi, J. Mater. Sci., 2009, 44, 4354-4359.

12 J. Tominaga, T. Nakano and N. Atoda, Appl. Phys. Lett., 1998, 73, 2078-2080.
13 H. Zou, X. Zhu, Y. Hu, Y. Sui, Y. Sun, J. Zhang, L. Zheng and Z. Song, J. Appl. Phys., 2016, 120, 245303.

14 T. Guo, S. Song, L. Li, X. Ji, C. Li, C. Xu, L. Shen, Y. Xue, B. Liu, Z. Song, M. Qi and S. Feng, Scr. Mater., 2017, 129, 56-60.

15 X. Zhu, Y. Hu, H. Zou, J. Zhang, Y. Sun, W. Wu, L. Yuan, L. Zhai, S. Song and Z. Song, Scr. Mater., 2016, 121, 66-69.

16 F. Xu, Y. Luo, M. Deng and Q. Shen, Eur. J. Org. Chem., 2003, 2003, 4728-4730.

17 C. Gao, H. Song, L. Hu, G. Pan, R. Qin, F. Wang, Q. Dai, L. Fan, L. Liu and H. Liu, J. Lumin., 2008, 128, 559-564.

18 X. Zhou, L. Wu, Z. Song, Y. Cheng, F. Rao, K. Ren, S. Song, B. Liu and S. Feng, Acta Mater., 2013, 61, 7324-7333.

19 X. Cheng, F. Mao, Z. Song, C. Peng and Y. Gong, Jpn. J. Appl. Phys., 2014, 53, 050304.

20 C. Ortiz and A. Blatter, Thin Solid Films, 1992, 218, 209-218.

21 Y. Hu, X. Feng, J. Zhai, T. Wen, T. Lai, S. Song and Z. Song, Scr. Mater., 2014, 93, 4-7.

22 W. Wu, Y. Hu, X. Zhu, Y. Sui, J. Xue, L. Yuan, S. Song and Z. Song, J. Mater. Sci.: Mater. Electron., 2015, 26, 9700-9706.

23 Z. Li, Y. Hu, T. Wen, J. Zhai and T. Lai, J. Appl. Phys., 2015, 117, 135703.

24 L. V. Pieterson, M. H. R. Lankhorst, M. V. Schijndel, A. E. T. Kuiper and J. H. J. Roosen, J. Appl. Phys., 2005, 97, 083520.

25 W. Wu, S. Chen, J. Zhai, X. Liu, T. Lai, S. Song and Z. Song, Appl. Phys. Lett., 2017, 110, 181906.

26 H. Zou, X. Zhu, Y. Hu, Y. Sui, L. Zheng, W. Wu, L. Zhai, J. Xue and Z. Song, J. Mater. Sci.: Mater. Electron., 2017, 28, 38063811.

27 Y. Hu, S. Li, T. Lai, S. Song, Z. Song and J. Zhai, J. Alloys Compd., 2013, 581, 515-518.

28 W. Fenfen, W. Long, K. Tao, S. Lin, H. Rong, Z. Jie and C. Guosheng, Appl. Phys. Lett., 2013, 103, 181908.

29 H. Zou, X. Zhu, Y. Hu, Y. Sun, L. Zheng, Y. Sui, S. Wu and Z. Song, J. Mater. Sci., 2017, 52, 5216-5222.

30 F. Wei, L. Wang, T. Kong, L. Shi, R. Huang, J. Zhang and G. Cheng, Appl. Phys. Lett., 2013, 103, 181908.

31 C. Peng, L. Wu, Z. Song, F. Rao, M. Zhu, X. Li, B. Liu, L. Cheng, S. Feng and P. Yang, Appl. Surf. Sci., 2011, 257, 10667-10670. 